\title{
Nivel de conocimiento de autoexploración mamaria en adolescentes
}

\section{Level of knowledge of breast self-examination in adolescents}

\author{
Michelle Pérez-Hernández y Enrique A. Sánchez-Valdivieso* \\ Departamento de Investigación y Cátedra de Oncología, Universidad Cristóbal Colón, Boca del Río, Veracruz, México
}

\begin{abstract}
Resumen
Introducción: Los métodos para la detección temprana del cáncer de mama incluyen la autoexploración y la mastografía. Objetivo: Determinar el nivel de conocimiento sobre la autoexploración mamaria desde la perspectiva de los adolescentes. Método: Estudio descriptivo, transversal, en estudiantes de bachillerato de ambos sexos. Se aplicó una encuesta sobre sus antecedentes y conocimientos acerca de la autoexploración mamaria, así mismo, se les realizó una prueba visual mediante una lámina ilustrada y se les interrogó sobre sus perspectivas. Resultados: Fueron incluidos 498 estudiantes: 325 (65.3\%) mujeres y 173 (34.7\%) varones. El promedio de edad fue de 16.0 años. El 92.2\% sí supo que es la autoexploración mamaria, el $21.7 \%$ respondió que médicos y enfermeras les dieron esa información, el $72.5 \%$ no se revisan, el 39.4\% de las mujeres y el $95.4 \%$ de los varones. La prueba visual demostró que la mayoría (73.1\%) se realiza la autoexploración pero no de una manera ordenada. Existe vergüenza y miedo por saber que se puede tener cáncer, lo cual es una limitante para la autoexploración. Conclusiones: Estos resultados difieren al incluir varones cuyo nivel de conocimiento es llamativamente menor; la proporción de participantes con conocimientos básicos es más elevada que lo reportado. Esto puede deberse a la edad o las características sociales. El papel de la salud comunitaria en brindar información para la detección temprana del cáncer de mama es crucial para disminuir la mortalidad por este cáncer.
\end{abstract}

Palabras clave: Cáncer. Mama. Autoexploración. Adolescente. Prevención.

\begin{abstract}
Introduction: Methods for early detection of breast cancer include self-examination and mastography. Objective: To determine the level of knowledge about breast self-examination from the perspective of adolescents. Method: Descriptive, transversal study, including high school students of both sexes. A survey about their background and knowledge about breast self-examination was applied; likewise, they underwent a visual test by an illustrated sheet and their perspective was questioned. Results: We included 498 high school students, 325 (65.3\%) women and 173 (34.7\%) males. The average age was 16.0 years. 92.2\% knew what breast self-examination is, $21.7 \%$ said that information was provided by doctors and nurses, in $72.5 \%$ their breast are not being reviewed, $39.4 \%$ of women and $95.4 \%$ of men; visual test showed that the majority $(73.1 \%)$ is doing the self-exploration but not in an orderly manner. There are shame and fear to know that they can have cancer, which are limiting factors for self-examination. Conclusions: These results differ by including men whose level of knowledge is remarkably lower; the proportion of participants with basic knowledge is greater than reported. This may be due to age or social characteristics. The role of the comunity health to provide information for early detection of breast cancer is crucial to reduce mortality from this cancer.
\end{abstract}

Key words: Cancer. Breast. Self-examination. Adolescent. Prevention.

\footnotetext{
Correspondencia:

Fecha de recepción: 15-09-2019

*Enrique A. Sánchez-Valdivieso

E-mail: easanchezv@gmail.com

Fecha de aceptación: 06-01-2020

DOI: 10.24875/RHJM.20000008

Disponible en internet: 10-03-2020 Rev Hosp Jua Mex. 2020;87(1):28-33

www.revistahospitaljuarez.com 1405-9622/@ 2020 Sociedad Médico-Quirúrgica del Hospital Juárez de México, A.C. Publicado por Permanyer. Este es un artículo open access bajo la licencia CC BY-NC-ND (http://creativecommons.org/licenses/by-nc-nd/4.0/).
} 


\section{Introducción}

El pronóstico del cáncer de mama (CM) depende en gran medida de la detección temprana, por lo que se ha insistido en la autoexploración mamaria (AEM) ${ }^{1}$. Identificar entre mamas normales y anormales, y el conocimiento de saber qué buscar y el tiempo apropiado resultan cruciales, ya que una masa mamaria indolora es ignorada por varios meses ${ }^{2}$. La detección temprana ha sido asociada con la disminución de la morbilidad y mortalidad por $\mathrm{CM}^{3}$. No existe suficiente evidencia para desaprobar la $\mathrm{AEM}^{4}$, siendo un método sencillo y no invasor adecuado para los más jóvenes ${ }^{5}$, por lo que resulta esencial educar sobre AEM como método de detección temprana ${ }^{6}$. La adolescencia es un periodo de transición durante el cual ocurren en el cuerpo cambios significativos; este periodo brinda la oportunidad de enseñar a delinear una conducta de salud para la adultez ${ }^{7,8}$. Existen pocos estudios sobre la práctica de la AEM entre estudiantes de educación secundaria y bachillerato ${ }^{7,8}$ porque las jóvenes no se consideran en riesgo de $\mathrm{CM}^{9}$. Sin embargo, del 10 al $15 \%$ de los casos de CM ocurrirán antes de los 30 años ${ }^{10,11}$.

Por su parte, el CM en hombres ha recibido poca atención. Los médicos no tienen la conciencia de la enfermedad y están frecuentemente mal informados sobre sus implicaciones físicas y psicológicas potenciales $^{12}$; sin embargo, al parecer, su incidencia está aumentando ${ }^{13,14}$. Enseñar temas acerca del $\mathrm{CM}$ al adolescente varón también estimulará una conducta positiva a largo plazo, que le puede llevar a buscar un examen clínico regular más tarde en su vida. Hasta el momento, no se han realizado estudios sobre el conocimiento del $\mathrm{CM}$ y la práctica de la AEM entre estudiantes varones de educación secundaria.

El objetivo de este estudio fue conocer el nivel de conocimiento sobre la AEM de adolescentes estudiantes de bachillerato de ambos sexos.

\section{Pacientes y métodos}

Estudio descriptivo transversal observacional de diseño metodológico mixto, cuantitativo y cualitativo, realizado como parte de la Cátedra de Oncología que favoreció la práctica en Salud Comunitaria sobre estudiantes de $2 .^{\circ}$ y $3 .^{\circ}$ año de bachillerato. El muestreo fue no probabilístico buscando obtener una muestra de 500 estudiantes de ambos sexos con edades entre los 15 y 18 años. Se excluyeron aquellos casados, embarazadas, con antecedente de tumor mamario o portadoras de prótesis mamarias. Se solicitó autorización de los padres y directivos escolares y firma del consentimiento informado. El instrumento fue un cuestionario validado en población mexicana ${ }^{15}$ modificado, dividido en varias secciones, de antecedentes personales, de ítems de conocimiento, y de perspectiva sobre la AEM, tipo Likert. Se realizó, además, una prueba visual con apoyo de una lámina ilustrada con cinco figuras, la cual contenía de manera desordenada los pasos de la AEM indicada en la Norma Oficial Mexicana NOM-041-SSA2-2002, y se pidió a los participantes que indicaran el orden correcto en que se debe realizar la AEM. Si el participante ordenó correctamente tres o más, se calificó como completa; y si ordenó menos de tres, se calificó como incompleta. Por último, agregamos una pregunta de corte cualitativo sobre las necesidades de los propios adolescentes para saber cómo autoexplorarse, cuyas respuestas se codificaron. Posterior a esto, se dio una plática informativa sobre factores de riesgo para CM y la técnica para realizar la autoexploración.

El análisis estadístico se realizó por estadística descriptiva, mediante frecuencias y porcentajes, medidas de tendencia central, medidas de dispersión, tomando el valor de $p<0.05$ para determinar significancia entre las variables. Para calcular estos parámetros se utilizó el paquete estadístico SPSS 24.0 (Chicago, III).

Tabla 1. Distribución de los estudiantes según las variables sociodemográficas y fuentes de información

\begin{tabular}{|c|c|c|c|}
\hline & Global & Mujeres & Hombres \\
\hline Sexo & $498(100 \%)$ & $325(65.3 \%)$ & $173(34.7 \%)$ \\
\hline Edad promedio & $\begin{array}{c}15.99( \pm \\
0.805)\end{array}$ & $15.95( \pm 0.800)$ & $16.07( \pm 0.811)$ \\
\hline Menarquia & & $11.45( \pm 1.197)$ & - \\
\hline $\begin{array}{l}\text { Fuentes de } \\
\text { información }\end{array}$ & $\begin{array}{l}\text { Global } \\
\text { n (\%) }\end{array}$ & $\begin{array}{c}\text { Mujeres } \\
\text { n (\%) }\end{array}$ & $\begin{array}{c}\text { Hombres } \\
\text { n (\%) }\end{array}$ \\
\hline $\begin{array}{l}\text { Medios } \\
\text { televisivos }\end{array}$ & $176(35.3)$ & $109(33.5)$ & $67(38.7)$ \\
\hline $\begin{array}{l}\text { Centro de } \\
\text { salud }\end{array}$ & $108(21.7)$ & $65(20.0)$ & $43(24.9)$ \\
\hline $\begin{array}{l}\text { Pláticas y } \\
\text { folletos }\end{array}$ & 68 (13.7) & $52(16.0)$ & $16(9.2)$ \\
\hline Escuela & 64 (12.9) & $39(12.0)$ & $25(14.5)$ \\
\hline $\begin{array}{l}\text { Familiar con } \\
\text { cáncer }\end{array}$ & $49(9.8)$ & $44(13.5)$ & $5(2.9)$ \\
\hline $\begin{array}{l}\text { Madre o } \\
\text { familia }\end{array}$ & $45(9.0)$ & $35(10.8)$ & $10(5.8)$ \\
\hline $\begin{array}{l}\text { Internet/toallas } \\
\text { femeninas }\end{array}$ & $36(7.2)$ & $25(7.7)$ & $11(6.4)$ \\
\hline
\end{tabular}




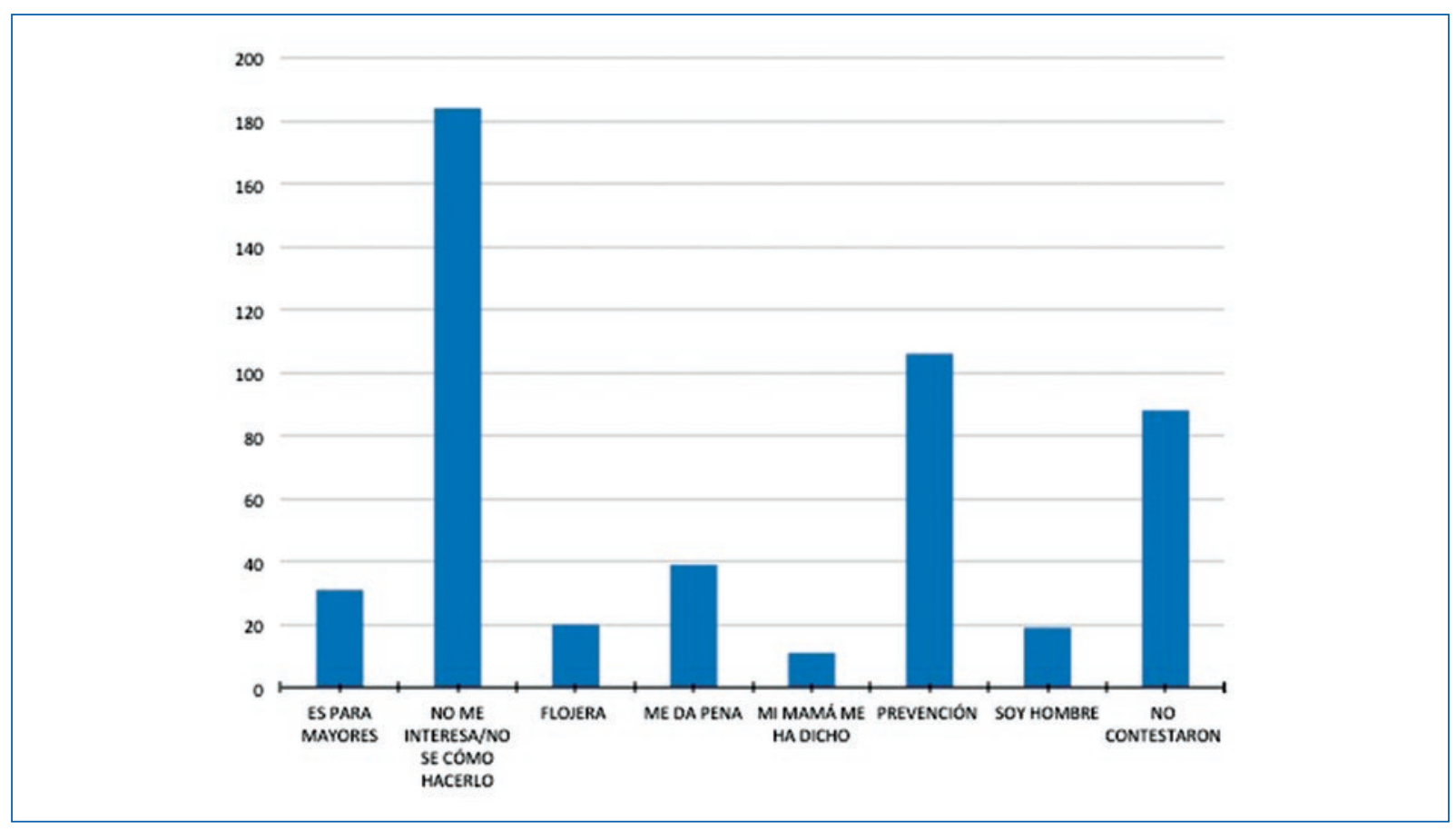

Figura 1. Razones por las que los estudiantes no se practicaban la autoexploración mamaria.

Este estudio fue aprobado por el Comité Institucional de Bioética.

\section{Resultados}

Se administraron los cuestionarios encuestando a 500 estudiantes. De estos, se descartaron dos por inconsistencias, por lo que la base del estudio fue de 498 estudiantes: 325 (65.3\%) del sexo femenino y $173(34.7 \%)$ del sexo masculino, con un promedio de edad de 16.0 años (Tabla 1). El 9.8\% manifestó tener familiares de primer grado con $\mathrm{CM}$. En mujeres, la edad media de la menarquia fue 11.5 años \pm 1.2 años (rango 9-15 años).

El 7.8\% (39 de los 498) no sabía qué es la AEM. La mayoría de los restantes se enteraron de la AEM por campañas de salud y medios de comunicación; proporciones menores dicen que por los mismos doctores y enfermeras $(21.7 \%)$ o por pláticas en la escuela $(12.8 \%)$; pocos $(7.2 \%)$ se enteran por información en los paquetes de toallas sanitarias.

El 27.5\% (137 encuestados) se revisa por cuestiones de prevención, revisiones médicas o por miedo porque algún familiar ya tuvo cáncer y no quieren que les pase lo mismo; 361 (72.5\%) no se revisan, $196(60.3 \%)$ de las 325 mujeres y 165 (95.4\%) de los 173 varones; el $39.4 \%$ de las mujeres no lo hace porque no saben, porque les da miedo o porque no se acuerdan; solo

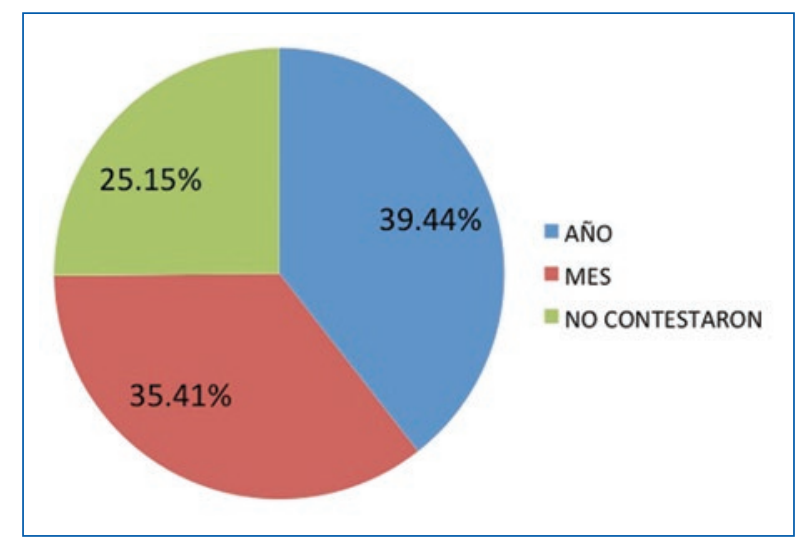

Figura 2. Periodicidad de la autoexploración mamaria según los estudiantes.

$8(4.6 \%)$ hombres se revisan los senos, los restantes tienen la idea de que, por ser hombres, no están en riesgo de CM (Fig. 1).

El $39.4 \%$ se realizan AEM anualmente, el $35.3 \%$ mensualmente y el resto ocasionalmente (Fig. 2). El $88 \%(n=438)$ no saben cuándo es el momento adecuado para realizar la revisión; el 12\% $(n=60)$ sí lo sabe, por visitas médicas con sus familiares. El 39\% ( $n=194)$ no sabe qué características deben tener las mamas, ya que no tienen una orientación adecuada por la falta de información; el $57 \%(n=284)$ sí sabe 


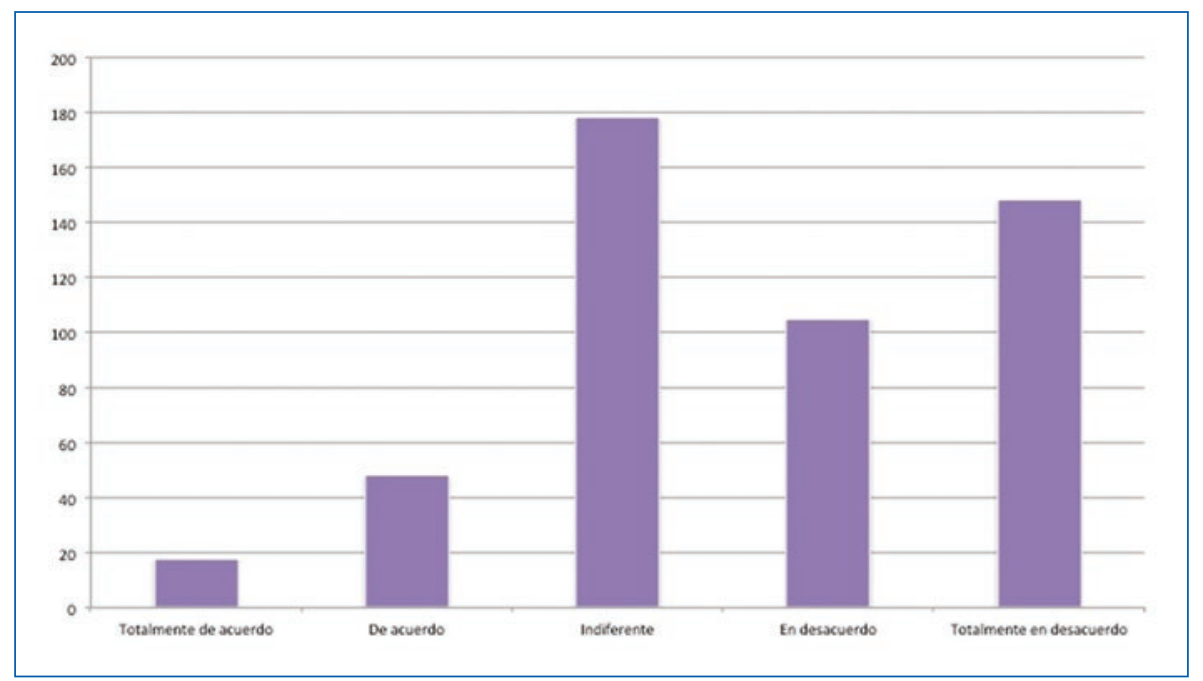

Figura 3. Respuestas a la pregunta: ¿te da vergüenza tocarte los senos?

por un familiar o conocido con $\mathrm{CM}$, o porque se encuentran bien informados; y 20 estudiantes (4\%) no respondieron.

El sentimiento que genera la palabra cáncer fue de tristeza ( $n=203 ; 40.8 \%)$, seguido de miedo $(n=113$; $22.7 \%$ ), ya que los enfermos con cáncer son sus propias abuelas o sus madres. Una proporción menor nos dijo que es una enfermedad mortal $(n=35 ; 7 \%)$.

El $50.6 \%(n=252)$ está de acuerdo en que la AEM es una manera fácil de detectar cáncer y que lo pueden hacer desde su hogar; un número pequeño $(n=4)$ estuvo en desacuerdo.

\section{Preguntas sobre percepción de la AEM}

- ¿La vergüenza es el principal motivo por el cual las mujeres no se revisan sus mamas?: La mayoría mujeres estuvo de acuerdo en que les da pena tocarlos.

- ¿Cualquier persona se puede realizar autoexploración de mamas?: El 75.7\% $(n=377)$ estuvo de acuerdo; 57 varones (11.4\%) estuvieron en desacuerdo porque dicen que no tendrán $\mathrm{CM}$.

- ¿Los senos son una parte del cuerpo que no se debe observar ni revisar?: El 89.4\% $(n=445)$ estuvo en desacuerdo, ya que es una parte importante a revisar.

- ¿Mujeres que presentaron alguna enfermedad en las mamas ya no deben realizar autoexploración?: El $55.2 \%$ ( $n=275$ ) estuvo en desacuerdo; aunque se haya tenido alguna enfermedad en las mamas, no se está exento de presentarla nuevamente, por lo que hay que estar alerta ante un posible cambio.
- ¿Se deben revisar los senos al menos una vez al mes?: El 82.9\% $(n=413)$ estuvo de acuerdo en revisarse al menos una vez al mes para disminuir el riesgo.

- ¿Te da vergüenza tocar tus senos?: Algunas mujeres indicaron que les da pena tocar sus senos, les da miedo, o ven raro el tocarse una parte íntima (individuos mal informados sobre el riesgo de $\mathrm{CM}$ y su prevención); el $35.7 \%(n=178)$ se mostró indiferente 0 estuvo en desacuerdo (Fig. 3).

- ¿Tienes miedo de tener CM?: El 54.6\% $(n=272)$ dijo que sí.

- ¿Qué necesitas saber para poder autoexplorarte?: La mayoría $(63.9 \%, \mathrm{n}=318)$ necesitaba recibir entrenamiento sobre la técnica para realizar la AEM (Fig. 4).

\section{Prueba visual}

El $73.1 \%(n=364)$ conoce el procedimiento, es decir, cuáles son los pasos a seguir para la autoexploración, pero no sabe cómo son los movimientos exactos para una buena revisión, ordenó menos de tres, por lo que la prueba se calificó como incompleta; la otra parte $(26.9 \%$; $n=134)$ no tiene idea de cómo empezar una AEM.

\section{Discusión}

La adolescencia ofrece oportunidades para la formación del comportamiento sanitario en la edad adulta ${ }^{7,8}$. Los adolescentes están iniciando a entender sus cuerpos, y enseñarles la AEM estimulará la conducta de buscar atención y examen clínico regular más adelante. En un estudio en jóvenes de $15-19$ años $^{16}$, solo el $27.8 \%$ de las encuestadas 


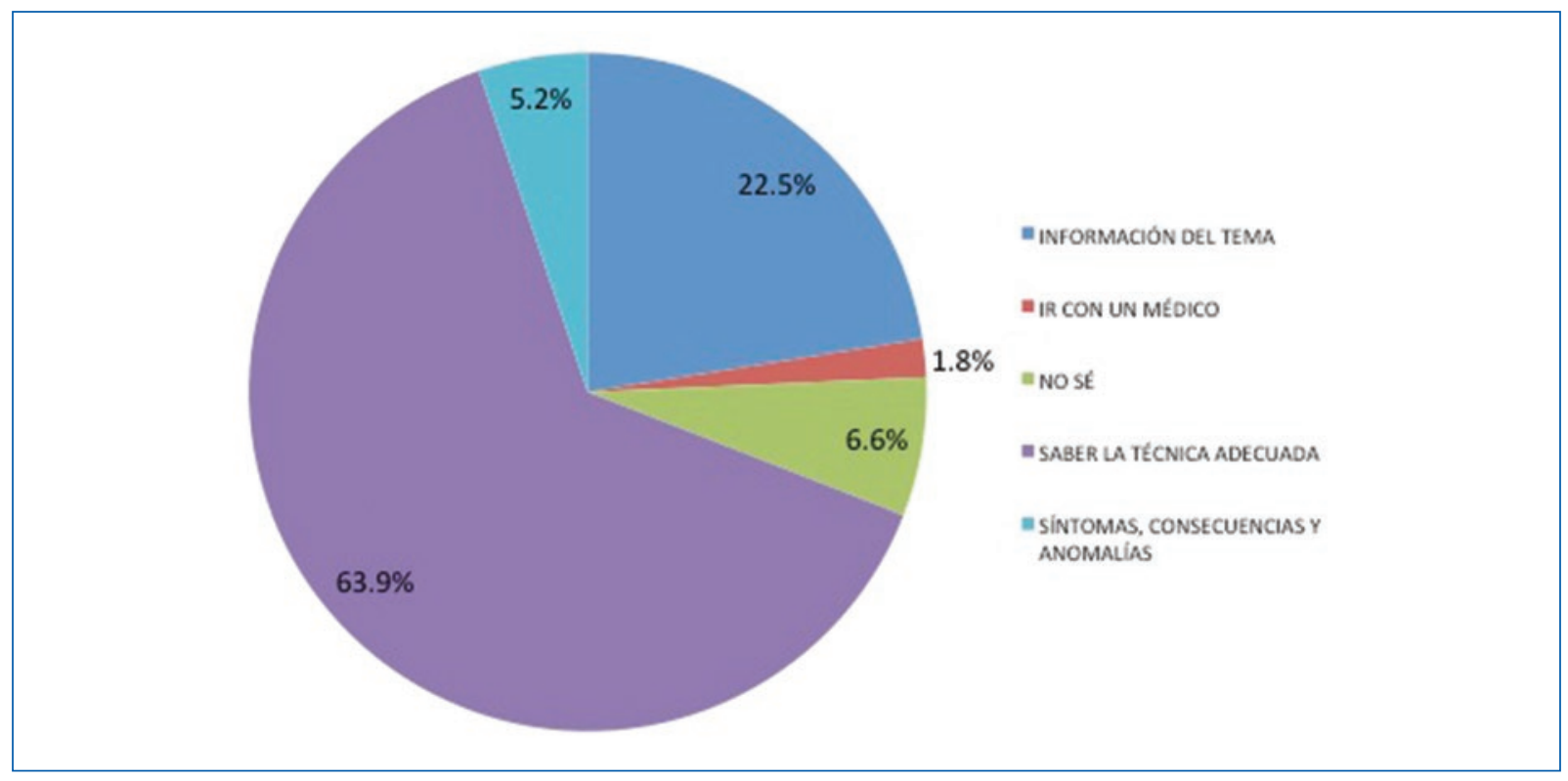

Figura 4. Necesidades detectadas en los estudiantes para practicar la autoexploración mamaria.

practicaba la AEM regularmente, con un nivel inadecuado de conocimiento de la AEM y de los factores de riesgo de CM. Los autores recalcan la importancia de los trabajadores sanitarios de impartir educación en salud mamaria a estudiantes de escuelas secundarias.

Otro estudio ${ }^{17}$ informó sobre el pobre conocimiento de factores de riesgo para $\mathrm{CM}$ y una pobre práctica de la $A E M$, y recomendó programas de intervención en jóvenes de secundaria dentro del plan curricular, así como talleres con padres y maestros.

Entre mujeres universitarias (el $96.8 \%$ solteras), el $19.6 \%$ realizaba la $\mathrm{AEM}^{18}$. Otro estudio detectó también un conocimiento inadecuado que afectó la práctica de la $\mathrm{AEM}^{19}$. A pesar de que el $61.7 \%$ reconoció que la $\mathrm{AEM}$ es un método de detección de $\mathrm{CM}$, el $70.8 \%$ de mujeres con edad media de 34.6 años no conocía cómo se realiza la $\mathrm{AEM}^{20}$. Entre mujeres nigerianas ${ }^{21}$, solo el $5.3 \%$ realizaba la AEM mensualmente, si bien, el 58.2\% había escuchado de ella. Las principales barreras para la AEM ${ }^{21}$ es desconocer cómo se realiza (47.6\%) y creer no estar en riesgo (47.6\%). Entre maestras etíopes ${ }^{22}$, el $73.1 \%$ nunca se había realizado una AEM y el $16.5 \%$ nunca había escuchado de ella. Se identificaron como predictores de autoexploración ${ }^{22}$ el conocimiento, la susceptibilidad percibida, la gravedad percibida y el beneficio neto percibido. En nuestras estudiantes, el nivel de conocimiento de la AEM es elevado, si bien el $39.4 \%$ de mujeres y un porcentaje mayor de varones no se revisa. El $72.5 \%$ (95.4\% de varones) no acostumbraba a realizarse la AEM, y la razón principal fue la percepción de no estar en riesgo. Existen muy pocos estudios en varones. Si bien es cierto que el CM masculino es poco frecuente, la incidencia es cada vez mayor. En un estudio entre universitarios ${ }^{23}$, con el $22 \%$ de hombres, la mitad de estudiantes mostró bajo nivel de conocimiento (<50\%). El $90.9 \%$ había escuchado de la AEM en la televisión, pero solo el $48.3 \%$ la practicaba. La razón más común era la falta de conocimiento de la técnica correcta ${ }^{23}$.

En un estudio en varones hacia la detección temprana de $\mathrm{CM}$, el $57.6 \%$ estaba consciente de la importancia de la AEM, y la fuente principal de información (43.4\%) era el médico ${ }^{24}$. Los autores sugieren campañas dirigidas a los esposos como agentes de cambio para comprometer a sus familias en acciones de detección ${ }^{24}$. La mayoría de varones universitarios $(72.2 \%$ estudiantes de medicina) percibe como baja su susceptibilidad a padecer $\mathrm{CM}^{25}$. Existe un déficit de información en varones sobre el cual aún se debe incidir. El CM en hombres ha recibido poca atención, no solo por parte del público sino también por los médicos, ya que carecen de consciencia de la enfermedad, a menudo mal informados sobre sus consecuencias físicas y psicológicas ${ }^{26}$. En un estudio en estudiantes universitarios ( $41 \%$ varones) se concluyó que la falta de conocimiento sobre CM remarca la necesidad de implementar programas de educación y prevención ${ }^{27}$. Por ello es crucial que los médicos y los educadores establezcan grupos entrenados en elevar el nivel de conocimiento en $\mathrm{CM}$. Entre mujeres, en el medio rural, solo el $47 \%$ se realizaba la autoexploración ${ }^{28}$. Ello puede 
deberse a que la población urbana tiene mayor acceso a información y a atención.

\section{Conclusiones}

Hay necesidad de conocimiento sobre la técnica adecuada de la AEM y de información, por lo que se necesita instruir a los adolescentes de educación secundaria y bachillerato para poder incidir en la disminución de la mortalidad por CM.

\section{Conflicto de intereses}

Los autores declaran no tener conflicto de intereses alguno.

\section{Financiamiento}

Los autores no recibieron patrocinio para llevar a cabo este artículo.

\section{Responsabilidades éticas}

Protección de personas y animales. Los autores declaran que los procedimientos seguidos se conformaron a las normas éticas del comité de experimentación humana responsable y de acuerdo con la Asociación Médica Mundial y la Declaración de Helsinki.

Confidencialidad de los datos. Los autores declaran que han seguido los protocolos de su centro de trabajo sobre la publicación de datos de pacientes.

Derecho a la privacidad y consentimiento informado. Los autores han obtenido el consentimiento informado de los pacientes y/o sujetos referidos en el artículo. Este documento obra en poder del autor de correspondencia.

\section{Bibliografía}

1. World Health Organization: Cancer. (Consultado en 2018 Julio 22). Disponible en: http://www.who.int/mediacentre/factsheets/fs297/en].

2. Ohene-Yeboah M, Amaning EP. The spectrum of complaints presented at a specialist breast clinic in Kumasi Ghana. Ghana Med J. 2008;42(3):110-12.

3. Mele N, Archer J, Pusch BD. Access to breast cancer screening services for women with disabilities. J Obstet Gynecol Neonat Nurs. 2005;34(A):453-64.

4. McCready T, Littlewood D, Jenkinson J. Breast self-examination and breast awareness: A literature review. J Clin Nurs. 2005;14(5):570-8.

5. Ewaid SH, Shanjar AM, Mahdi RH. Knowledge and practice of breast self-examination among sample of women in Shatra/Dhi-Qar/lraq. Alexandria J Med. 2018;54(4):315-7.
6. Quang Tuyen DQ, Viet Dung T, Van Dong H, Trung Kien T, Thanh Huong T. Breast Self-Examination: Knowledge and Practice Among Female Textile Workers in Vietnam. Cancer Control. 2019;26:1-7.

7. Ahenkorah Fondjo L, Owusu-Afriyie O, Asamoah Sakyi O, Addo Wiafe A Amankwaa B, Acheampong E, et al. Comparative Assessment of Knowledge, Attitudes, and Practice of Breast Self-Examination among Female Secondary and Tertiary School Students in Ghana. Int J Breast Cancer. 2018:2018:1-10.

8. Milaat WA. Knowledge of secondary school female students on breast cancer and breast self examination in Jeddah, Saudi Arabia. East Mediterr Health J. 2000;6:338-44.

9. Johnsona N, Dickson-Swifta V. It usually happens in older women': Young women's perceptions about breast cancer. Health Education Journal. 2008;67(4):243-57.

10. Adebamowo CA, Ajayi OO. Breast cancer in Nigeria. West Afr J Med. 2000;19:179-91.

11. Okobia MN, Bunker $\mathrm{CH}$, Okonofua FE, Osime U. Knowledge, attitude and practice of Nigerian women towards breast cancer: a cross sectional study. World J Surg Oncol. 2006;4:11-6.

12. Robinson JD, Metoyer KPJr, Bhayani N. Breast cancer in men: a need for psychological intervention. J Clin Psychol Med Settings. 2008;15:134-9.

13. Stang A, Thomssen C. Decline in breast cancer incidence in the United States: what about male breast cancer? Breast Cancer Res Treat. 2008;112:595-6.

14. Peate I. Caring for men with breast cancer: causes, symptoms and treatment. Br J Nurs. 2001;10:975-81.

15. Yépez-RamírezD, de la Rosa AG, Guerrero-Albarrán C, Gómez-MartínezJM. Autoexploración mamaria: conocimiento y perspectiva en mujeres. Rev Enferm Inst Mex Seguro Soc. 2012;20(2):79-84.

16. Che CC, Coomarasamy JD, Suppayah B. Perception of breast health amongst Malaysian female adolescents. Asian Pac J Cancer Prev. 2014;15(17):7175-80.

17. Isara AR, Ojedokun $\mathrm{Cl}$. Knowledge of breast cancer and practice of breast self examination among female senior secondary school students in Abuja, Nigeria. J Prev Med Hyg. 2011;52:186-90.

18. Akhtari-Zavare M, Juni MH, Ismail IZ, Said SM, Latiff LA. Health Beliefs and Breast Self-Examination among Undergraduate Female Students in Public Universities in Klang Valley, Malaysia. Asian Pac J Cancer Prev. 2015;16(9):4019-23.

19. Nde FP, Assob JCN, Kwenti TE, Njunda AL, Tainenbe TRG. Knowledge, attitude and practice of breast self-examination among female undergraduate students in the University of Buea. BMC Res Notes. 2015;8:43-8.

20. Oladimeji KE, Tsoka-Gwegweni JM, Igbodekwe FC, Twomey M, Akolo C, Balarabe HS, et al. Knowledge and Beliefs of Breast Self-Examination and Breast Cancer among Market Women in Ibadan, South West, Nigeria. PLoS One. 2015:10(11):e0140904.

21. Amoran OE, Toyobo OO. Predictors of breast self-examination as cancer prevention practice among women of reproductive age-group in a rural town in Nigeria. Niger Med J. 2015;56(3):185-9.

22. Birhane N, Mamo A, Girma E, Asfaw S. Predictors of breast self-examination among female teachers in Ethiopia using health belief model. Arch Public Health. 2015;73(1):39-46.

23. Alwan NA, Al-Attar WM, Eliessa RA, Madfaie ZA, Tawfeeq FN. Knowledge, attitude and practice regarding breast cancer and breast self-examination among a sample of the educated population in Iraq. East Mediterr Health J. 2012;18(4):337-45.

24. Al-Amoudi SM, Abduljabbar HS. Men's knowledge and attitude towards breast cancer in Saudi Arabia. A cross-sectional study. Saudi Med J. 2012;33(5):547-50.

25. Al-Naggar RA, Al-Naggar DH. Perceptions and opinions about male breast cancer and male breast self-examination: A qualitative study. Asian Pacific J Cancer Prev. 2012;13:243-6.

26. Midding $E$, Halbach SM, Kowalski $C$, Weber $R$, Würstlein $R$, Ernstmann N. Men With a "Woman's Disease": Stigmatization of Male Breast Cancer Patients-A Mixed Methods Analysis. Am J Men's Health. 2018;12(6):2194-207.

27. Ayed A, Eqtait F, Harazneh L, Fashafsheh I, Nazzal S, Talahmeh B, et al. Breast Self-Examination in Terms of Knowledge, Attitude, and Practice among Nursing Students of Arab American University/Jenin. J Educ Pract. 2015;6(4):37-47.

28. Torres EE, García MGI, Marín GM, Andrade DBE. Aprendizaje significativo de mujeres entre 20 y 59 años ante la autoexploración de mama. Rev Enferm IMSS. 2004;12(3):131-5. 